\title{
Reduced description in the reaction kinetics
}

\author{
A.N. Gorban ${ }^{\mathrm{a}}$, I.V. Karlin ${ }^{\mathrm{b}, *}$, V.B. Zmievskii ${ }^{\mathrm{c}}$, S.V. Dymova ${ }^{\mathrm{d}}$ \\ ${ }^{a}$ Institute of Computational Modeling RAS, Krasnoyarsk 660036, Russia \\ ${ }^{\mathrm{b}}$ Department of Materials, ETH Zürich, Institute of Polymers, CH-8092 Zürich, Switzerland \\ ${ }^{\mathrm{c}}$ EPFL, Fluid Mechanics Laboratory, CH-1024 Lausanne, Switzerland \\ dinstitute of Computational Modeling RAS, Krasnoyarsk 660036, Russia
}

Received 25 May 1999

\begin{abstract}
Models of complex reactions in thermodynamically isolated systems often demonstrate evolution towards low-dimensional manifolds in the phase space. For this class of models, we suggest a direct method to construct such manifolds, and thereby to reduce the effective dimension of the problem. The approach realizes the invariance principle of the reduced description, it is based on iterations rather than on a small parameter expansion, it leads to tractable linear problems, and is consistent with thermodynamic requirements. The approach is tested with a model of catalytic reaction. (c) 2000 Elsevier Science B.V. All rights reserved.
\end{abstract}

PACS: $05.20 . \mathrm{Dd} ; 82.20 . \mathrm{Mj}$

Keywords: Reaction kinetics; Reduced description; Invariant manifolds

\section{Introduction}

The goal of nonequilibrium statistical physics is the understanding of how a system with many degrees of freedom acquires a description with a few degrees of freedom. This should lead to reliable methods of extracting the macroscopic description from a detailed microscopic description.

Meanwhile, this general problem is still far from the final solution, it is reasonable to study simplified models, where, on the one hand, a detailed description is accessible to numerics, on the other hand, analytical methods designed to the solution of problems in real systems can be tested.

\footnotetext{
${ }^{*}$ Corresponding author. Fax: +41-1-632-10-76.

E-mail address: ikarlin@ifp.mat.ethz.ch (I.V. Karlin)
} 
In this paper we address a well-known class of finite-dimensional systems known from the theory of reaction kinetics. These are equations governing the relaxation in perfectly stirred isolated chemically active mixtures. Dissipative properties of such systems are characterized with a global convex Lyapunov function $G$ which implements the second law of thermodynamics: as the time $t$ tends to infinity, the system reaches the unique equilibrium state while in the course of the transition the Lyapunov function decreases monotonically.

While the limiting behavior of the dissipative systems just described is certainly very simple, there are still interesting questions to be asked about. One of these questions is closely related to the above general problem of nonequilibrium statistical physics. Indeed, evidence of numerical integration of such systems often demonstrates that the relaxation has a certain geometrical structure in the phase space. Namely, typical individual trajectories tend to manifolds of lower dimension, and further proceed to the equilibrium essentially along these manifolds. Thus, such systems demonstrate a dimensional reduction, and therefore establish a more macroscopic description after some time since the beginning of the relaxation.

In this paper, we develop a general method of constructing the reduced description for dissipative systems of reaction kinetics. Our approach is based on the method of invariant manifold which has been suggested in Ref. [1]. Its realization for a generic dissipative systems has been discussed in paper [2]. This method has been applied to a set of specific problems of classical kinetic theory based on the Boltzmann equation [2-5]. The goal of the present study is twofold. The first goal is to verify the method of invariant manifold on a representative class of nonlinear dissipative systems. The second goal concerns a more practical issue, namely, the problem of an effective description of complex reactions. For example, the reaction $\mathrm{O}_{2}+2 \mathrm{H}_{2}=2 \mathrm{H}_{2} \mathrm{O}$ is complex: Besides $\mathrm{O}_{2}, \mathrm{H}_{2}$ and $\mathrm{H}_{2} \mathrm{O}$, intermediate products should be taken into account [such as $\mathrm{O}, \mathrm{H}, \mathrm{OH}, \mathrm{O}_{2} \mathrm{H}, \mathrm{O}_{2} \mathrm{H}_{2}$, etc.], and the detailed mechanism of the reaction includes 30 elementary steps, or even more [6,7]. Though the systems we address in this paper are finite dimensional, they may be sufficiently large, and are able to create difficulties for numerical studies. Therefore, methods to reduce the original detailed description are needed to make the analysis more tractable.

There are two intuitive ideas behind our approach, and we shall now discuss them informally. Objects to be considered below are manifolds (hypersurfaces) $\boldsymbol{\Omega}$ in the phase space of the reaction kinetic systems (the phase space is usually a convex polyhedron in a finite-dimensional real space). The 'ideal' picture of the reduced description we have in mind is as follows: A typical phase trajectory $c(t)$, where $t$ is the time, and $c$ is an element of the phase space, consists of two pronounced segments. The first segment connects the beginning of the trajectory $c(0)$ with a certain point $c\left(t_{1}\right)$ on the manifold $\boldsymbol{\Omega}$. The second segment belongs to $\boldsymbol{\Omega}$, and connects the point $\boldsymbol{c}\left(t_{1}\right)$ with the equilibrium $\boldsymbol{c}^{\mathrm{eq}}=\boldsymbol{c}(\infty), \boldsymbol{c}^{\mathrm{eq}} \in \boldsymbol{\Omega}$. Thus, the manifolds appearing in our ideal picture are 'patterns' formed by the segments of individual trajectories.

There are two important features behind this ideal picture. The first feature is the invariance of the manifold $\boldsymbol{\Omega}$ : Once the individual trajectory has reached $\boldsymbol{\Omega}$, it does 
not leave $\boldsymbol{\Omega}$ anymore. The second feature is the projecting: The phase points outside $\boldsymbol{\Omega}$ will be projected onto $\boldsymbol{\Omega}$. Furthermore, the dissipativity of the system provides an additional information about this ideal picture: Regardless of what happens on the manifold $\boldsymbol{\Omega}$, the function $G$ was decreasing along each individual trajectory before it reached $\boldsymbol{\Omega}$.

This ideal picture of the decomposition of motions is certainly too exaggerated, but it is a useful guide to extract the reduced description. The main advantage is that it is completely geometrical, allows for a direct and fairly simple formalization, and makes it possible to apply rapidly convergent iteration methods of solution.

The paper is organized as follows. In the Section 2, we review the reaction kinetics, and introduce the method of invariant manifold in the way appropriate to this class of nonequilibrium systems. In the Sections 3 and 4 we give some details on the two relatively independent parts of the method, the thermodynamic projector, and the iterations for solving the invariance equation. We also introduce a general symmetric linearization procedure for the invariance equation, and discuss its relevance to the picture of decomposition of motions. In the Section 5, these two procedures are combined into an unique algorithm. In the Section 6, we demonstrate an example of computations for a model catalytic reaction. Finally, results are discussed in the Section 7.

\section{Manifolds of reduced description}

\subsection{Outline of the dissipative reaction kinetics}

We start with a review of the reaction kinetics (for details see e.g. Refs. [6,7]). Let us consider an isolated system with $n$ chemical species $A_{1}, \ldots, A_{n}$, participating in a complex reaction. The complex reaction is represented by the following stoichiometric mechanism:

$$
\alpha_{s 1} A_{1}+\cdots+\alpha_{s n} A_{n} \rightleftharpoons \beta_{s 1} A_{1}+\cdots+\beta_{s n} A_{n},
$$

where the index $s=1, \ldots, r$ enumerates the reaction steps, and where integers $\alpha_{s i}$ and $\beta_{s i}$ are stoichiometric coefficients. For each reaction step $s$, we introduce $n$-component vectors $\boldsymbol{\alpha}_{s}$ and $\boldsymbol{\beta}_{s}$ with components $\alpha_{s i}$ and $\beta_{s i}$. Notation $\boldsymbol{\gamma}_{s}$ stands for the vector with integer components $\gamma_{s i}=\beta_{s i}-\alpha_{s i}$ (the stoichiometric vector). We adopt an abbreviated notation for the standard scalar product of the $n$-component vectors:

$$
\langle\boldsymbol{x}, \boldsymbol{y}\rangle=\sum_{i=1}^{n} x_{i} y_{i} .
$$

The system is described by $n$-component concentration vector $\boldsymbol{c}$, where the component $c_{i} \geqslant 0$ represents the concentration of the specie $A_{i}$. Conservation laws impose linear constraints on admissible vectors $c$ :

$$
\left\langle\boldsymbol{b}_{i}, \boldsymbol{c}\right\rangle=B_{i}, \quad i=1, \ldots, l,
$$


where $\boldsymbol{b}_{i}$ are fixed and linearly independent vectors, and $B_{i}$ are given scalars. Let us denote as $\boldsymbol{B}$ the set of vectors which satisfy the conservation laws (2):

$$
\boldsymbol{B}=\left\{\boldsymbol{c} \mid\left\langle\boldsymbol{b}_{1}, \boldsymbol{c}\right\rangle=B_{1}, \ldots,\left\langle\boldsymbol{b}_{l}, \boldsymbol{c}\right\rangle=B_{l}\right\} .
$$

The phase space $\boldsymbol{V}$ of the system is the intersection of the set of $n$-dimensional vectors with nonnegative components, with the set $\boldsymbol{B}$, and $\operatorname{dim} \boldsymbol{V}=d=n-l$. In the sequel, we term a vector $\boldsymbol{c} \in \boldsymbol{V}$ the state of the system. In addition, we assume that each of the conservation laws is supported by each elementary reaction step, that is

$$
\left\langle\boldsymbol{\gamma}_{s}, \boldsymbol{b}_{i}\right\rangle=0
$$

for each pair of vectors $\gamma_{s}$ and $\boldsymbol{b}_{i}$.

Reaction kinetic equations describe variations of the states in time. Given the stoichiometric mechanism (1), the reaction kinetic equations reads

$$
\dot{\boldsymbol{c}}=\boldsymbol{J}(\boldsymbol{c}), \quad \boldsymbol{J}(\boldsymbol{c})=\sum_{s=1}^{r} \boldsymbol{\gamma}_{s} W_{s}(\boldsymbol{c}),
$$

where dot denotes the time derivative, and $W_{s}$ is the reaction rate function of the step $s$. In particular, the mass action law suggests the polynomial form of the reaction rates:

$$
W_{s}=k_{s}^{+} \prod_{i=1}^{n} c_{i}^{\alpha_{i}}-k_{s}^{-} \prod_{i=1}^{n} c_{i}^{\beta_{i}},
$$

where $k_{s}^{+}$and $k_{s}^{-}$are the constants of the direct and of the inverse reactions rates of the $s$ th reaction step. The phase space $\boldsymbol{V}$ is positive-invariant of the system (4): if $\boldsymbol{c}(0) \in \boldsymbol{V}$, then $\boldsymbol{c}(t) \in \boldsymbol{V}$ for all the times $t>0$.

In the sequel, we assume that the kinetic equation (4) describes an evolution towards the unique equilibrium state $\boldsymbol{c}^{\mathrm{eq}}$ in the interior of the phase space $\boldsymbol{V}$. Furthermore, we assume that there exists a strictly convex function $G(c)$ which decreases monotonically in time due to Eq. (4):

$$
\dot{G}=\langle\nabla G(\boldsymbol{c}), \boldsymbol{J}(\boldsymbol{c})\rangle \leqslant 0 .
$$

Here $\nabla G$ is the vector of partial derivatives $\partial G / \partial c_{i}$, and the convexity assumes that the $n \times n$ matrices

$$
\boldsymbol{H}_{\boldsymbol{c}}=\left\|\partial^{2} G(\boldsymbol{c}) / \partial c_{i} \partial c_{j}\right\|
$$

are positive definite for all $\boldsymbol{c} \in \boldsymbol{V}$. In addition, we assume that matrices (7) are invertible if $c$ is taken in the interior of the phase space.

The function $G$ is the Lyapunov function of the system (4), and $c^{\text {eq }}$ is the point of global minimum of the function $G$ in the phase space $\boldsymbol{V}$. Otherwise stated, the manifold of equilibrium states $\boldsymbol{c}^{\mathrm{eq}}\left(B_{1}, \ldots, B_{l}\right)$ is the solution to the variational problem,

$$
G \rightarrow \min \text { for }\left\langle\boldsymbol{b}_{i}, \boldsymbol{c}\right\rangle=B_{i}, \quad i=1, \ldots, l .
$$

For each fixed value of the conserved quantities $B_{i}$, the solution is unique. In many cases, however, it is convenient to consider the whole equilibrium manifold, keeping the conserved quantities as parameters. 
For example, for perfect systems in a constant volume under a constant temperature, the Lyapunov function $G$ reads

$$
G=\sum_{i=1}^{n} c_{i}\left[\ln \left(c_{i} / c_{i}^{\mathrm{eq}}\right)-1\right]
$$

Finally, we recall an important generalization of the mass action law (5), known as the Marcelin-De Donder kinetic function [8-10]. Within this approach, the functions $W_{s}$ are constructed as follows: For a given strictly convex function $G$, and for a given stoichiometric mechanism (1), we define the gain $(+)$ and the loss $(-)$ rates of the sth step,

$$
\begin{aligned}
& W_{s}^{+}=\varphi_{s}^{+} \exp \left[\left\langle\nabla G, \boldsymbol{\alpha}_{s}\right\rangle\right], \\
& W_{s}^{-}=\varphi_{s}^{-} \exp \left[\left\langle\nabla G, \boldsymbol{\beta}_{s}\right\rangle\right],
\end{aligned}
$$

where $\varphi_{s}^{ \pm}>0$ are kinetic factors. The Marcelin-De Donder kinetic function reads: $W_{s}=W_{s}^{+}-W_{s}^{-}$, and the right hand-side of the kinetic equation (4) becomes

$$
\boldsymbol{J}=\sum_{s=1}^{r} \gamma_{s}\left\{\varphi_{s}^{+} \exp \left[\left\langle\nabla G, \boldsymbol{\alpha}_{s}\right\rangle\right]-\varphi_{s}^{-} \exp \left[\left\langle\nabla G, \beta_{s}\right\rangle\right]\right\} .
$$

For the Marcelin-De Donder reaction rate (10), the dissipation inequality (6) reads

$$
\dot{G}=\sum_{s=1}^{r}\left[\left\langle\nabla G, \boldsymbol{\beta}_{s}\right\rangle-\left\langle\nabla G, \boldsymbol{\alpha}_{s}\right\rangle\right]\left\{\varphi_{s}^{+} \exp \left[\left\langle\nabla G, \boldsymbol{\alpha}_{s}\right\rangle\right]-\varphi_{s}^{-} \exp \left[\left\langle\nabla G, \boldsymbol{\beta}_{s}\right\rangle\right]\right\} \leqslant 0 .
$$

The kinetic factors $\varphi_{s}^{ \pm}$should satisfy certain conditions in order to make valid the dissipation inequality (12). A well-known sufficient condition is the detail balance:

$$
\varphi_{s}^{+}=\varphi_{s}^{-}
$$

other sufficient conditions are discussed in detail elsewhere [6,7,11]. For the function $G$ of the form (9), the Marcelin-De Donder equation casts into the more familiar mass action law form (5). In the sequel we adopt the Marcelin-De Donder formulation of the dissipative reaction kinetics.

\subsection{Outline of the reduced description}

In many cases, dynamics of the $d$-dimensional system (4) leads to a manifold of a lower dimension. Intuitively, a typical phase trajectory behaves as follows: Given the initial state $c(0)$ at $t=0$, and after some period of time, the trajectory comes close to some low-dimensional manifold $\boldsymbol{\Omega}$, and after that proceeds towards the equilibrium essentially along this manifold. The goal is to construct this manifold.

The starting point of our approach is based on a formulation of the two main requirements: 
(i) Dynamic invariance: The manifold $\boldsymbol{\Omega}$ should be invariant under the dynamics of the originating system (4): If $\boldsymbol{c}(0) \in \boldsymbol{\Omega}$, then $\boldsymbol{c}(t) \in \boldsymbol{\Omega}$ for each $t>0$.

(ii) Thermodynamic consistency of the reduced dynamics: Let some (not obligatory invariant) manifold $\boldsymbol{\Omega}$ is considered as a manifold of reduced description. We should define a set of linear operators, $\boldsymbol{P}_{\boldsymbol{c}}$, labeled by the states $\boldsymbol{c} \in \boldsymbol{\Omega}$, which project the vectors $\boldsymbol{J}(\boldsymbol{c}), \boldsymbol{c} \in \boldsymbol{\Omega}$ onto the tangent bundle of the manifold $\boldsymbol{\Omega}$, thereby generating the induced vector field, $\boldsymbol{P}_{\boldsymbol{c}} \boldsymbol{J}(\boldsymbol{c}), \boldsymbol{c} \in \boldsymbol{\Omega}$. This induced vector field on the tangent bundle of the manifold $\boldsymbol{\Omega}$ is identified with the reduced dynamics along the manifold $\boldsymbol{\Omega}$. The thermodynamicity requirement for this induced vector field reads

$$
\left\langle\nabla G(c), \boldsymbol{P}_{c} \boldsymbol{J}(\boldsymbol{c})\right\rangle \leqslant 0 \quad \text { for each } \boldsymbol{c} \in \boldsymbol{\Omega} \text {. }
$$

In order to meet these requirements, the method of invariant manifold suggests two complementary procedures:

(i) To treat the condition of dynamic invariance as an equation, and to solve it iteratively by a Newton method. This procedure is geometric in its nature, and it does not use the time dependence and small parameters.

(ii) Given an approximate manifold of reduced description, to construct the projector satisfying condition (14) in a way which does not depend on the vector field $\boldsymbol{J}$. We shall now outline both these procedures starting with the second. The solution consists, in the first place, in formulating the thermodynamic condition which should be met by the projectors $\boldsymbol{P}_{\boldsymbol{c}}$ : For each $\boldsymbol{c} \in \boldsymbol{\Omega}$, let us consider the linear functional

$$
M_{c}^{*}(\boldsymbol{x})=\langle\nabla G(\boldsymbol{c}), \boldsymbol{x}\rangle \text {. }
$$

Then the thermodynamic condition for the projectors reads

$$
\operatorname{ker} \boldsymbol{P}_{\boldsymbol{c}} \subseteq \operatorname{ker} M_{c}^{*} \quad \text { for each } \boldsymbol{c} \in \boldsymbol{\Omega}
$$

Here $\operatorname{ker} \boldsymbol{P}_{\boldsymbol{c}}$ is the null space of the projector, and $\operatorname{ker} M_{\boldsymbol{c}}^{*}$ is the hyperplane orthogonal to the vector $M_{c}^{*}$. It has been shown in Ref. [2] that condition (16) is the necessary and sufficient condition to establish the thermodynamic induce vector field on the given manifold $\boldsymbol{\Omega}$ for all possible dissipative vector fields $\boldsymbol{J}$ simultaneously.

Let us now turn to the requirement of invariance. By a definition, the manifold $\boldsymbol{\Omega}$ is invariant with respect to the vector field $\boldsymbol{J}$ if and only if the following equality is true:

$$
[1-\boldsymbol{P}] \boldsymbol{J}(\boldsymbol{c})=0 \quad \text { for each } \boldsymbol{c} \in \boldsymbol{\Omega} .
$$

In this expression $\boldsymbol{P}$ is an arbitrary projector on the tangent bundle of the manifold $\boldsymbol{\Omega}$. It has been suggested to consider condition (17) as an equation to be solved iteratively starting with some appropriate initial manifold.

Iterations for the invariance equation (17) are considered in the Section 4. The next section presents construction of the thermodynamic projector using a specific parameterization of manifolds. 


\section{Thermodynamic projector}

\subsection{Thermodynamic parameterization}

In this section, $\boldsymbol{\Omega}$ denotes a generic $p$-dimensional manifold. First, it should be mentioned that any parameterization of $\boldsymbol{\Omega}$ generates a certain projector, and thereby a certain reduced dynamics [2]. Indeed, let us consider a set of $m$ independent functionals $M(c)=\left\{M_{1}(c), \ldots, M_{p}(c)\right\}$, and let us assume that they form a coordinate system on $\boldsymbol{\Omega}$ in such a way that $\boldsymbol{\Omega}=\boldsymbol{c}(M)$, where $\boldsymbol{c}(M)$ is a vector function of the parameters $M_{1}, \ldots, M_{p}$. Then the projector associated with this parameterization reads

$$
\boldsymbol{P}_{\boldsymbol{c}(M)} \boldsymbol{x}=\sum_{i, j=1}^{p} \frac{\partial \boldsymbol{c}(M)}{\partial M_{i}} N_{i j}^{-1}(M)\left\langle\left.\nabla M_{j}\right|_{\boldsymbol{c}(M)}, \boldsymbol{x}\right\rangle,
$$

where $N_{i j}^{-1}$ is the inverse to the $p \times p$ matrix:

$$
N(M)=\left\|\left\langle\nabla M_{i}, \partial c / \partial M_{j}\right\rangle\right\| .
$$

This somewhat involved notation is intended to stress that projector (18) is dictated by the choice of the parameterization. Subsequently, the induced vector field of the reduced dynamics is found by applying projectors (18) on the vectors $\boldsymbol{J}[\boldsymbol{c}(M)]$, thereby inducing the reduced dynamics in terms of the parameters $M$ as follows:

$$
\dot{M}_{i}=\sum_{j=1}^{p} N_{i j}^{-1}(M)\left\langle\left.\nabla M_{j}\right|_{\boldsymbol{c}(M)}, \boldsymbol{J}[\boldsymbol{c}(M)]\right\rangle .
$$

Depending on the choice of the parameterization, dynamic equations (20) are (or are not) consistent with the thermodynamic requirement (14). The thermodynamic parameterization makes use of condition (16) in order to establish the thermodynamic projector. Specializing to case (18), let us consider the linear functionals,

$$
\left.D M_{i}\right|_{\boldsymbol{c}(M)}(\boldsymbol{x})=\left\langle\left.\nabla M_{i}\right|_{\boldsymbol{c}(M)}, \boldsymbol{x}\right\rangle .
$$

Then condition (16) takes the form:

$$
\left.\bigcap_{i=1}^{p} \operatorname{ker} D M_{i}\right|_{c(M)} \subseteq \operatorname{ker} M_{c(M)}^{*},
$$

that is, the intersection of null spaces of the functionals (21) should belong to the null space of the differential of the Lyapunov function $G$, in each point of the manifold $\boldsymbol{\Omega}$.

In practice, in order to construct the thermodynamic parameterization, we take the following set of functionals in each point $c$ of the manifold $\boldsymbol{\Omega}$ :

$$
\begin{aligned}
& M_{1}(\boldsymbol{x})=M_{\boldsymbol{c}}^{*}(\boldsymbol{x}), \quad \boldsymbol{c} \in \boldsymbol{\Omega}, \\
& M_{i}(\boldsymbol{x})=\left\langle\boldsymbol{m}_{i}, \boldsymbol{x}\right\rangle, \quad i=2, \ldots, p .
\end{aligned}
$$

It is required that vectors $\boldsymbol{\nabla} G(\boldsymbol{c}), \boldsymbol{m}_{2}, \ldots, \boldsymbol{m}_{p}$ are linearly independent in each state $\boldsymbol{c} \in \boldsymbol{\Omega}$. Inclusion of functionals (15) as a part of system (23) implies the thermodynamic 
condition (22). Also, any linear combination of the parameter set (23) will meet the thermodynamicity requirement.

It is important to notice here that the thermodynamic condition is satisfied whatsoever the functionals $M_{2}, \ldots, M_{p}$ are. This is very convenient for it gives an opportunity to take into account the conserved quantities correctly. The manifolds we are going to deal with should be consistent with the conservation laws (2). While the explicit characterization of the phase space $\boldsymbol{V}$ is a problem on its own, in practice, it is customary to work in the $n$-dimensional space while keeping constraints (2) explicitly on each step of the construction. For this technical reason, it is convinient to consider manifolds of the dimension $p>l$, where $l$ is the number of conservation laws, in the $n$-dimensional space rather than in the phase space $\boldsymbol{V}$. The thermodynamic parameterization is then concordant also with the conservation laws if $l$ of the linear functionals (23b) are identified with the conservation laws. In the sequel, only projectors consistent with conservation laws are considered.

Very frequently, the manifold $\boldsymbol{\Omega}$ is represented as a $p$-parametric family $c\left(a_{1}, \ldots, a_{p}\right)$, where $a_{i}$ are coordinates on the manifold. The thermodynamic re-parameterization in terms of variables (23) suggests a representation of the coordinates $a_{i}$ in terms of $M_{c}^{*}, M_{2}, \ldots, M_{p}(23)$. While the explicit construction of these functions may be a formidable task, we notice that the construction of the thermodynamic projector of the form (18) and of the dynamic equations (20) is relatively easy because only the derivatives $\partial c / \partial M_{i}$ enter these expressions. This point was discussed in a detail in Refs. [1,2]. Here refer to an example considered below [Section 6, Eq. (51)].

\subsection{Decomposition of motions: thermodynamics}

Finally, let us discuss how the thermodynamic projector is related to the decomposition of motions. Assuming that the decomposition of motions near the manifold $\boldsymbol{\Omega}$ is true indeed, let us consider states which were initially close enough to the manifold $\boldsymbol{\Omega}$. Even without knowing the details about the evolution of the states towards $\boldsymbol{\Omega}$, we know that the Lyapunov function $G$ was decreasing in the course of this evolution. Let us consider a set of states $\boldsymbol{U}_{\boldsymbol{c}}$ which contains all those vectors $\boldsymbol{c}^{\prime}$ that have arrived (in other words, have been projected) into the point $\boldsymbol{c} \in \boldsymbol{\Omega}$. Then we observe that the state $c$ furnishes the minimum of the function $G$ on the set $\boldsymbol{U}_{\boldsymbol{c}}$. If a state $\boldsymbol{c}^{\prime} \in \boldsymbol{U}_{\boldsymbol{c}}$, and if it deviates small enough from the state $c$ so that the linear approximation is valid, then $\boldsymbol{c}^{\prime}$ belongs to the affine hyperplane

$$
\Gamma_{c}=c+\operatorname{ker} M_{c}^{*}, \quad c \in \boldsymbol{\Omega} .
$$

This hyperplane actually participates in condition (16). The consideration was entiteled 'thermodynamic' [1] because it describes the states $\boldsymbol{c} \in \boldsymbol{\Omega}$ as points of minimum of the function $G$ over the corresponding hyperplanes (24). 


\section{Corrections}

\subsection{Preliminary discussion}

The thermodynamic projector is needed to induce the dynamics on a given manifold in such a way that the dissipation inequality (14) holds. Coming back to the issue of constructing corrections, we should stress that the projector participating in the invariance condition (17) is arbitrary. It is convinient to make use of this point: When Eq. (17) is solved iteratively, the projector may be kept nonthermodynamic unless the induced dynamics is explicitly needed.

Let us assume that we have chosen the initial manifold, $\boldsymbol{\Omega}_{0}$, together with the associated projector $\boldsymbol{P}_{0}$, as the first approximation to the desired manifold of reduced description. Though the choice of the initial approximation $\boldsymbol{\Omega}_{0}$ depends on the specific problem, it is often reasonable to consider quasi-equilibrium or quasi-stationary approximations [they will be discussed below in the Sections 5 and 6]. In most cases, the manifold $\boldsymbol{\Omega}_{0}$ is not an invariant manifold. This means that $\boldsymbol{\Omega}_{0}$ does not satisfy the invariance condition (17):

$$
\boldsymbol{\Delta}_{0}=\left[1-\boldsymbol{P}_{0}\right] \boldsymbol{J}\left(\boldsymbol{c}_{0}\right) \neq 0 \text { for some } \boldsymbol{c}_{0} \in \boldsymbol{\Omega}_{0} .
$$

Therefore, we seek a correction $\boldsymbol{c}_{1}=\boldsymbol{c}_{0}+\delta \boldsymbol{c}$. Substituting $\boldsymbol{P}=\boldsymbol{P}_{0}$ and $\boldsymbol{c}=\boldsymbol{c}_{0}+\delta \boldsymbol{c}$ into the invariance equation (17), and after the linearization in $\delta \boldsymbol{c}$, we derive the following linear equation:

$$
\left[1-\boldsymbol{P}_{0}\right]\left[\boldsymbol{J}\left(\boldsymbol{c}_{0}\right)+\boldsymbol{L}_{\boldsymbol{c}_{0}} \delta \boldsymbol{c}\right]=0,
$$

where $\boldsymbol{L}_{c_{0}}$ is the matrix of first derivatives of the vector function $\boldsymbol{J}$, computed in the state $\boldsymbol{c}_{0} \in \boldsymbol{\Omega}_{0}$. The system of linear algebraic equations (26) should be supplied with the additional condition.

$$
\boldsymbol{P}_{0} \delta c=0 .
$$

In order to illustrate the nature of the Eq. (26), let us consider the case of linear manifolds for linear systems. Let a linear evolution equation is given in the finite-dimensional real space: $\dot{\boldsymbol{c}}=\boldsymbol{L} \boldsymbol{c}$, where $\boldsymbol{L}$ is negatively definite symmetric matrix with a simple spectrum. Let us further assume the quadratic Lyapunov function, $G(\boldsymbol{c})=\langle\boldsymbol{c}, \boldsymbol{c}\rangle$. The manifolds we consider are lines, $\boldsymbol{l}(a)=a \boldsymbol{e}$, where $\boldsymbol{e}$ is the unit vector, and $a$ is a scalar. The invariance equation for such manifolds reads: $\boldsymbol{e}\langle\boldsymbol{e}, \boldsymbol{L} \boldsymbol{e}\rangle-\boldsymbol{L} \boldsymbol{e}=0$, and is simply a form of the eigenvalue problem for the operator $\boldsymbol{L}$. Solutions to the latter equation are eigenvectors $\boldsymbol{e}_{i}$, corresponding to eigenvalues $\lambda_{i}$.

Assume that we have chosen a line, $\boldsymbol{l}_{0}=a \boldsymbol{e}_{0}$, defined by the unit vector $\boldsymbol{e}_{0}$, and that $e_{0}$ is not an eigenvector of $\boldsymbol{L}$. We seek another line, $\boldsymbol{l}_{1}=a \boldsymbol{e}_{1}$, where $\boldsymbol{e}_{1}$ is another unit vector, $\boldsymbol{e}_{1}=\boldsymbol{y}_{1} /\left\|\boldsymbol{y}_{1}\right\|, \boldsymbol{y}_{1}=\boldsymbol{e}_{0}+\delta \boldsymbol{y}$. The additional condition (27) now reads: $\left\langle\delta \boldsymbol{y}, \boldsymbol{e}_{0}\right\rangle=0$. Then the Eq. (26) becomes $\left[1-\boldsymbol{e}_{0}\left\langle\boldsymbol{e}_{0}, \cdot\right\rangle\right] L\left[\boldsymbol{e}_{0}+\delta \boldsymbol{y}\right]=0$. Subject to the additional condition, the unique solution is as follows: $\boldsymbol{e}_{0}+\delta \boldsymbol{y}=\left\langle\boldsymbol{e}_{0}, \boldsymbol{L}^{-1} \boldsymbol{e}_{0}\right\rangle^{-1} \boldsymbol{L}^{-1} \boldsymbol{e}_{0}$. Rewriting the latter expression in the eigenbasis of $\boldsymbol{L}$, we have: $\boldsymbol{e}_{0}+\delta \boldsymbol{y} \propto \sum_{i} \lambda_{i}^{-1} \boldsymbol{e}_{i}\left\langle\boldsymbol{e}_{i}, \boldsymbol{e}_{0}\right\rangle$. 
The leading term in this sum corresponds to the eigenvalue with the minimal absolute value. The example indicates that the method of linearization (26) seeks the direction of the slowest relaxation. For this reason, method (26) can be recognized as the basis of an iterative method for constructing the manifolds of slow motions.

For the nonlinear systems, the matrix $\boldsymbol{L}_{c_{0}}$ in the Eq. (26) depends nontrivially on $\boldsymbol{c}_{0}$. In this case system (26) requires a further specification which will be done now.

\subsection{Symmetric linearization}

The invariance condition (17) supports a lot of invariant manifolds, and not all of them are relevant to the reduced description (for example, any individual trajectory is itself an invariant manifold). This should be carefully taken into account when deriving a relevant equation for the correction in the states of the initial manifold $\boldsymbol{\Omega}_{0}$ which are located far from equilibrium. This point concerns the procedure of the linearization of the vector field $\boldsymbol{J}$, appearing in Eq. (26). We shall return to the explicit form of the Marcelin-De Donder kinetic function (10). Let $c$ is an arbitrary fixed element of the phase space. The linearization of the vector function $\boldsymbol{J}$ (11) about $\boldsymbol{c}$ may be written $\boldsymbol{J}(\boldsymbol{c}+\delta \boldsymbol{c}) \approx \boldsymbol{J}(\boldsymbol{c})+\boldsymbol{L}_{\boldsymbol{c}} \delta \boldsymbol{c}$ where the linear operator $\boldsymbol{L}_{\boldsymbol{c}}$ acts as follows:

$$
\boldsymbol{L}_{\boldsymbol{c}} \boldsymbol{x}=\sum_{s=1}^{r} \gamma_{s}\left[W_{s}^{+}(\boldsymbol{c})\left\langle\boldsymbol{\alpha}_{s}, \boldsymbol{H}_{\boldsymbol{c}} \boldsymbol{x}\right\rangle-W_{s}^{-}(\boldsymbol{c})\left\langle\boldsymbol{\beta}_{s}, \boldsymbol{H}_{\boldsymbol{c}} \boldsymbol{x}\right\rangle\right] .
$$

Here $\boldsymbol{H}_{\boldsymbol{c}}$ is the matrix of second derivatives of the function $G$ in the state $\boldsymbol{c}$ [see Eq. (7)]. The matrix $\boldsymbol{L}_{\boldsymbol{c}}$ in the Eq. (28) can be decomposed as follows:

$$
\boldsymbol{L}_{c}=\boldsymbol{L}_{c}^{\prime}+\boldsymbol{L}_{c}^{\prime \prime}
$$

Matrices $\boldsymbol{L}_{\boldsymbol{c}}^{\prime}$ and $\boldsymbol{L}_{\boldsymbol{c}}^{\prime \prime}$ act as follows:

$$
\begin{aligned}
& \boldsymbol{L}_{\boldsymbol{c}}^{\prime} \boldsymbol{x}=-\frac{1}{2} \sum_{s=1}^{r}\left[W_{s}^{+}(\boldsymbol{c})+W_{s}^{-}(\boldsymbol{c})\right] \gamma_{s}\left\langle\gamma_{s}, \boldsymbol{H}_{\boldsymbol{c}} \boldsymbol{x}\right\rangle, \\
& \boldsymbol{L}_{\boldsymbol{c}}^{\prime \prime} \boldsymbol{x}=\frac{1}{2} \sum_{s=1}^{r}\left[W_{s}^{+}(\boldsymbol{c})-W_{s}^{-}(\boldsymbol{c})\right] \gamma_{s}\left\langle\boldsymbol{\alpha}_{s}+\boldsymbol{\beta}_{s}, \boldsymbol{H}_{\boldsymbol{c}} \boldsymbol{x}\right\rangle .
\end{aligned}
$$

In order to highlight some features of this decomposition, let us introduce another scalar product, $\left\langle\langle\boldsymbol{x} \mid \boldsymbol{y}\rangle_{\boldsymbol{c}}\right.$, generated by the positive-definite matrix $\boldsymbol{H}_{\boldsymbol{c}}$,

$$
\langle\langle\boldsymbol{x} \mid \boldsymbol{y}\rangle\rangle_{c}=\left\langle\boldsymbol{x}, \boldsymbol{H}_{c} \boldsymbol{y}\right\rangle \text {. }
$$

The following properties of the matrix $\boldsymbol{L}_{c}^{\prime}$ are verified immediately:

(i) The matrix $\boldsymbol{L}_{\boldsymbol{c}}^{\prime}$ is symmetric in the scalar product (31):

$$
\left\langle\left\langle\boldsymbol{x}\left|\boldsymbol{L}_{c}^{\prime}\right| \boldsymbol{y}\right\rangle_{c}=\left\langle\left\langle\boldsymbol{y}\left|\boldsymbol{L}_{c}^{\prime}\right| \boldsymbol{x}\right\rangle\right\rangle_{c} .\right.
$$

(ii) The matrix $\boldsymbol{L}_{c}^{\prime}$ is nonpositive definite in the scalar product (31):

$$
\left\langle\left\langle\boldsymbol{x}\left|\boldsymbol{L}_{\boldsymbol{c}}^{\prime}\right| \boldsymbol{x}\right\rangle\right\rangle_{c} \leqslant 0 .
$$


(iii) The null space of the matrix $\boldsymbol{L}_{\boldsymbol{c}}^{\prime}$ is the linear envelope of the vectors $\boldsymbol{H}_{\boldsymbol{c}}^{-1} \boldsymbol{b}_{i}$ representing the complete system of conservation laws:

$$
\operatorname{ker} \boldsymbol{L}_{\boldsymbol{c}}^{\prime}=\operatorname{Lin}\left\{\boldsymbol{H}_{\boldsymbol{c}}^{-1} \boldsymbol{b}_{i}, i=1, \ldots, l\right\}
$$

(iv) If $\boldsymbol{c}=\boldsymbol{c}^{\mathrm{eq}}$, then $W_{s}^{+}\left(\boldsymbol{c}^{\mathrm{eq}}\right)=W_{s}^{-}\left(\boldsymbol{c}^{\mathrm{eq}}\right)$, and

$$
\boldsymbol{L}_{c^{\mathrm{eq}}}^{\prime}=\boldsymbol{L}_{c^{\mathrm{eq}}} .
$$

Thus, the decomposition Eq. (29) splits the matrix $\boldsymbol{L}_{\boldsymbol{c}}$ in two parts: one part, Eq. (30a) is symmetric and nonpositive definite, while the other part, Eq. (30b), vanishes in the equilibrium. The decomposition Eq. (29) explicitly takes into account the MarcelinDe Donder form of the kinetic function. For other dissipative systems, decomposition (29) is possible as soon as the relevant kinetic operator is written in a gain-loss form [for instance, this is straightforward for the Boltzmann collision operator].

In the sequel, we shall make use of the properties of the operator $\boldsymbol{L}_{\boldsymbol{c}}^{\prime}$ (30a) for constructing the dynamic correction by extending the picture of the decomposition of motions.

\subsection{Decomposition of motions: kinetics}

The assumption about the existence of the decomposition of motions near the manifold of reduced description $\boldsymbol{\Omega}$ has led to the thermodynamic specifications of the states $\boldsymbol{c} \in \boldsymbol{\Omega}$. This was accomplished in the Section 3.2, where the thermodynamic projector was backed by an appropriate variational formulation, and this has helped us to establish the induced dynamics consistent with the dissipation property. Another important feature of the decomposition of motions is that the states $\boldsymbol{c} \in \boldsymbol{\Omega}$ can be specified kinetically. Indeed, let us 'freeze' again the dynamics along the manifold $\boldsymbol{\Omega}$, and lets us consider the process of relaxation towards a state $\boldsymbol{c} \in \boldsymbol{\Omega}$. Dynamics towards the state $\boldsymbol{c} \in \boldsymbol{\Omega}$ is described with the linear operator $\boldsymbol{L}_{\boldsymbol{c}}$. That is, for the sates $\boldsymbol{c}+\delta \boldsymbol{c} \in \Gamma_{\boldsymbol{c}_{0}}$, where $\Gamma_{c_{0}}$ is the hyperplane (24), we have a relaxation equation, $\dot{\delta} \boldsymbol{c}=\boldsymbol{L}_{c} \delta c$. This relaxation equation has the quadratic Lyapunov function, $\delta G=\langle\langle\delta \boldsymbol{c} \mid \delta \boldsymbol{c}\rangle\rangle_{\boldsymbol{c}}$, that is, $\left\langle\left\langle\delta \boldsymbol{c}\left|\boldsymbol{L}_{\boldsymbol{c}}\right| \delta \boldsymbol{c}\right\rangle_{\boldsymbol{c}} \leqslant 0\right.$.

Let us consider now the manifold $\boldsymbol{\Omega}_{0}$ which is not the invariant manifold of the reduced description but, by our assumption, is located close to it. Consider a state $\boldsymbol{c}_{0} \in \boldsymbol{\Omega}_{0}$, and the states $\boldsymbol{c}_{0}+\delta \boldsymbol{c}$ close to it. Further, let us consider an equation

$$
\dot{\delta} \boldsymbol{c}=\boldsymbol{L}_{\boldsymbol{c}_{0}}^{\prime} \delta \boldsymbol{c} .
$$

Due to the properties of the operator $\boldsymbol{L}_{\boldsymbol{c}_{0}}^{\prime}(30 \mathrm{a})$, this equation can be regarded as a model of the assumed true relaxation equation near the true manifold of the reduced description. For this reason, we shall use the symmetric operator $\boldsymbol{L}_{\boldsymbol{c}}^{\prime}$ (30a) instead of the linear operator $\boldsymbol{L}_{\boldsymbol{c}}$ when constructing the corrections. It should be stressed here that the symmetric linearization (30a) is by no means unique. A different way of deriving the symmetric linearization will be discussed in the Section 7 . 


\subsection{Symmetric iteration}

Let the manifold $\boldsymbol{\Omega}_{0}$ and the corresponding projector $\boldsymbol{P}_{0}$ are the initial approximation to the invariant manifold of the reduced description. The dynamic correction $\boldsymbol{c}_{1}=\boldsymbol{c}_{0}+\delta \boldsymbol{c}$ is found upon solving the following system of linear algebraic equations:

$$
\begin{aligned}
& {\left[1-\boldsymbol{P}_{0}\right]\left[\boldsymbol{J}\left(\boldsymbol{c}_{0}\right)+\boldsymbol{L}_{\boldsymbol{c}_{0}}^{\prime} \delta \boldsymbol{c}\right]=0,} \\
& \boldsymbol{P}_{0} \delta \boldsymbol{c}=0 .
\end{aligned}
$$

Here $\boldsymbol{L}_{\boldsymbol{c}_{0}}^{\prime}$ is matrix (30a) taken in the states on the manifold $\boldsymbol{\Omega}_{0}$. An important technical point here is that the linear system (34a) always has the unique solution for any choice of the manifold $\boldsymbol{\Omega}$. This point is crucial since it guarantees the opportunity of carrying out the correction process for arbitrary number of steps.

\section{The method of invariant manifold}

We shall now combine together the two procedures discussed above. The resulting method of invariant manifold intends to seek iteratively the reduced description, starting with an initial approximation.

(i) Initialization: In order to start the procedure, it is required to choose the initial manifold $\boldsymbol{\Omega}_{0}$, and to derive corresponding thermodynamic projector $\boldsymbol{P}_{0}$. In the majority of cases, initial manifolds are available in two different ways. The first case are the quasi-equilibrium manifolds. Let us consider concentrations of the $k$ species $A_{1}, \ldots, A_{k}$ for relevant macroscopic variables (for example, concentrations of all stable products of the complex reaction). The macroscopic parameters are $M_{i}=c_{i}=\left\langle\boldsymbol{m}_{i}, \boldsymbol{c}\right\rangle$, where $\boldsymbol{m}_{i}$ is the unit vector corresponding to the specie $A_{i}$. The quasi-equilibrium manifold, $\boldsymbol{c}_{0}\left(M_{1}, \ldots, M_{k}, B_{1}, \ldots, B_{l}\right)$, compatible with the conservation laws, is the solution to the variational problem:

$$
\begin{aligned}
& G \rightarrow \min ,\left\langle\boldsymbol{m}_{i}, \boldsymbol{c}\right\rangle=c_{i}, \quad i=1, \ldots, k, \\
& \left\langle\boldsymbol{b}_{j}, \boldsymbol{c}\right\rangle=B_{j}, \quad j=1, \ldots, l .
\end{aligned}
$$

In the case of quasi-equilibrium approximation, the corresponding thermodynamic projector can be written most straightforwardly in terms of the variables $M_{i}$ :

$$
\boldsymbol{P}_{0} \boldsymbol{x}=\sum_{i=1}^{k} \frac{\partial \boldsymbol{c}_{0}}{\partial c_{i}}\left\langle\boldsymbol{m}_{i}, \boldsymbol{x}\right\rangle+\sum_{i=1}^{l} \frac{\partial \boldsymbol{c}_{0}}{\partial B_{i}}\left\langle\boldsymbol{b}_{i}, \boldsymbol{x}\right\rangle .
$$

For quasi-equilibrium manifolds, a re-parameterization with set (23) is not necessary [2].

The second source of initial approximations are quasi-stationary manifolds. They are derived by setting to zero a subset of reaction rates to arrive at the equations, $W_{s}(\boldsymbol{c})=0, s=s_{1}, \ldots, s_{k}$. Solution to these equations gives the quasi-stationary manifold, parameterized with the concentrations $c_{s_{1}}, \ldots, c_{s_{k}}$. Unlike the quasi-equilibrium case, the 
quasi-stationary manifolds must be re-parameterized in order to construct the thermodynamic projector. In the sequel we shall consider an example of both the quasiequilibrium and the quasi-stationary initial manifolds.

(ii) Corrections: Iterations are organized in accord with the rule: If $\boldsymbol{c}_{m}$ is the $m$ th approximation to the invariant manifold, then the correction $\boldsymbol{c}_{m+1}=\boldsymbol{c}_{m}+\delta \boldsymbol{c}$ is found from the linear algebraic equations,

$$
\begin{aligned}
& {\left[1-\boldsymbol{P}_{m}\right]\left(\boldsymbol{J}\left(\boldsymbol{c}_{m}\right)+\boldsymbol{L}_{\boldsymbol{c}_{m}}^{\prime} \delta \boldsymbol{c}\right)=0,} \\
& \boldsymbol{P}_{m} \delta \boldsymbol{c}=0 .
\end{aligned}
$$

Here $\boldsymbol{L}_{\boldsymbol{c}_{m}}^{\prime}$ is the symmetric matrix (30a) evaluated at the $m$ th approximation. The non-thermodynamic projector $\boldsymbol{P}_{m}$ is taken as follows:

$$
\boldsymbol{P}_{m} \boldsymbol{x}=\sum_{i=1}^{k} \frac{\partial \boldsymbol{c}_{m}}{\partial c_{i}}\left\langle\boldsymbol{m}_{i}, \boldsymbol{x}\right\rangle+\sum_{i=1}^{l} \frac{\partial \boldsymbol{c}_{m}}{\partial B_{i}}\left\langle\boldsymbol{b}_{i}, \boldsymbol{x}\right\rangle .
$$

(iii) Dynamics: Dynamics on the $m$ th manifold is obtained with the thermodynamic re-parameterization.

In the next section we shall test how this all works.

\section{Example: The two-step catalytic reaction}

Here we consider a two-step four-component reaction with one catalyst $A_{2}$ :

$$
A_{1}+A_{2} \rightleftharpoons A_{3} \rightleftharpoons A_{2}+A_{4} \text {. }
$$

We assume the Lyapunov function of the form (9), $G=\sum_{i=1}^{4} c_{i}\left[\ln \left(c_{i} / c_{i}^{\mathrm{eq}}\right)-1\right]$. The kinetic equation for the four-component vector of concentrations, $\boldsymbol{c}=\left(c_{1}, c_{2}, c_{3}, c_{4}\right)$, has the form

$$
\dot{\boldsymbol{c}}=\gamma_{1} W_{1}+\gamma_{2} W_{2} .
$$

Here $\gamma_{1,2}$ are stoichiometric vectors,

$$
\gamma_{1}=(-1,-1,1,0), \quad \gamma_{2}=(0,1,-1,1)
$$

while functions $W_{1,2}$ are reaction rates:

$$
\begin{aligned}
& W_{1}=k_{1}^{+} c_{1} c_{2}-k_{1}^{-} c_{3}, \\
& W_{2}=k_{2}^{+} c_{3}-k_{2}^{-} c_{2} c_{4} .
\end{aligned}
$$

Here $k_{1,2}^{ \pm}$are reaction rate constants. The system under consideration has two conservation laws,

$$
\begin{aligned}
& c_{1}+c_{3}+c_{4}=B_{1}, \\
& c_{2}+c_{3}=B_{2},
\end{aligned}
$$


or $\left\langle\boldsymbol{b}_{1,2}, \boldsymbol{c}\right\rangle=B_{1,2}$, where $\boldsymbol{b}_{1}=(1,0,1,1)$ and $\boldsymbol{b}_{2}=(0,1,1,0)$. The nonlinear system (40) is effectively two-dimensional, and we consider a one-dimensional reduced description.

In the first test, we have chosen the concentration of the specie $A_{1}$ as the variable of reduced description: $M=c_{1}$, and $c_{1}=\langle\boldsymbol{m}, \boldsymbol{c}\rangle$, where $\boldsymbol{m}=(1,0,0,0)$. The initial manifold $\boldsymbol{c}_{0}(M)$ was taken as the quasi-equilibrium approximation, i.e. the vector function $\boldsymbol{c}_{0}$ is the solution to the problem:

$$
G \rightarrow \min \text { for }\langle\boldsymbol{m}, \boldsymbol{c}\rangle=c_{1}, \quad\left\langle\boldsymbol{b}_{1}, \boldsymbol{c}\right\rangle=B_{1}, \quad\left\langle\boldsymbol{b}_{2}, \boldsymbol{c}\right\rangle=B_{2} .
$$

The solution to problem (44) reads

$$
\begin{aligned}
& c_{01}=c_{1}, \\
& c_{02}=B_{2}-\phi\left(c_{1}\right), \\
& c_{03}=\phi\left(c_{1}\right), \\
& c_{04}=B_{1}-c_{1}-\phi\left(c_{1}\right), \\
& \phi(M)=A\left(c_{1}\right)-\sqrt{A^{2}\left(c_{1}\right)-B_{2}\left(B_{1}-c_{1}\right)}, \\
& A\left(c_{1}\right)=\frac{B_{2}\left(B_{1}-c_{1}^{\mathrm{eq}}\right)+c_{3}^{\mathrm{eq}}\left(c_{1}^{\mathrm{eq}}+c_{3}^{\mathrm{eq}}-c_{1}\right)}{2 c_{3}^{\mathrm{eq}}} .
\end{aligned}
$$

Notice that this manifold is also the quasi-stationary manifold of the second reaction step, i.e. $W_{2}\left(c_{0}\right)=0$. The thermodynamic projector associated with the manifold (45) reads:

$$
\boldsymbol{P}_{0} \boldsymbol{x}=\frac{\partial \boldsymbol{c}_{0}}{\partial c_{1}}\langle\boldsymbol{m}, \boldsymbol{x}\rangle+\frac{\partial \boldsymbol{c}_{0}}{\partial B_{1}}\left\langle\boldsymbol{b}_{1}, \boldsymbol{x}\right\rangle+\frac{\partial \boldsymbol{c}_{0}}{\partial B_{2}}\left\langle\boldsymbol{b}_{2}, \boldsymbol{x}\right\rangle .
$$

Computing $\Delta_{0}=\left[1-\boldsymbol{P}_{0}\right] \boldsymbol{J}\left(\boldsymbol{c}_{0}\right)$ we find that inequality (25) takes place, and thus the manifold $\boldsymbol{c}_{0}$ is not invariant. The first correction, $\boldsymbol{c}_{1}=\boldsymbol{c}_{0}+\delta \boldsymbol{c}$, is found from the linear algebraic system (34a)

$$
\begin{aligned}
& \left(1-\boldsymbol{P}_{0}\right) \boldsymbol{L}_{0}^{\prime} \delta \boldsymbol{c}=-\left[1-\boldsymbol{P}_{0}\right] \boldsymbol{J}\left(\boldsymbol{c}_{0}\right), \\
& \delta c_{1}=0, \\
& \delta c_{1}+\delta c_{3}+\delta c_{4}=0, \\
& \delta c_{3}+\delta c_{2}=0,
\end{aligned}
$$

where the symmetric $4 \times 4$ matrix $\boldsymbol{L}_{0}^{\prime}$ has the form (we write 0 instead of $\boldsymbol{c}_{0}$ in the subscript in order to simplify notations):

$$
L_{0, k l}^{\prime}=-\gamma_{1 k} \frac{W_{1}^{+}\left(\boldsymbol{c}_{0}\right)+W_{1}^{-}\left(\boldsymbol{c}_{0}\right)}{2} \frac{\gamma_{1 l}}{c_{0 l}}-\gamma_{2 k} \frac{W_{2}^{+}\left(\boldsymbol{c}_{0}\right)+W_{2}^{-}\left(\boldsymbol{c}_{0}\right)}{2} \frac{\gamma_{2 l}}{c_{0 l}} .
$$

The explicit solution $\boldsymbol{c}_{1}\left(c_{1}, B_{1}, B_{2}\right)$ to the linear system (47) is easily found, and we do not reproduce it here. The process was iterated. On the $k+1$ iteration, the following projector $\boldsymbol{P}_{k}$ was used:

$$
\boldsymbol{P}_{k} \boldsymbol{x}=\frac{\partial \boldsymbol{c}_{k}}{\partial c_{1}}\langle\boldsymbol{m}, \boldsymbol{x}\rangle+\frac{\partial \boldsymbol{c}_{k}}{\partial B_{1}}\left\langle\boldsymbol{b}_{1}, \boldsymbol{x}\right\rangle+\frac{\partial \boldsymbol{c}_{k}}{\partial B_{2}}\left\langle\boldsymbol{b}_{2}, \boldsymbol{x}\right\rangle .
$$


Notice that projector $\boldsymbol{P}_{k}(50)$ is the thermodynamic projector only if $k=0$. As we have already mentioned it above, in the process of finding the corrections to the manifold, the non-thermodynamic projectors are allowed. The linear equation at the $k+1$ iteration is thus obtained by replacing $\boldsymbol{c}_{0}, \boldsymbol{P}_{0}$, and $\boldsymbol{L}_{0}^{\prime}$ with $\boldsymbol{c}_{k}, \boldsymbol{P}_{k}$, and $\boldsymbol{L}_{k}^{\prime}$ in all the entries of Eqs. (47) and (49).

Once the manifold $c_{k}$ was obtained on the $k$ th iteration, we derived the corresponding dynamics by introducing the thermodynamic parameterization (and the corresponding thermodynamic projector) with the help of function (23a). The resulting dynamic equation for the variable $c_{1}$ in the $k$ th approximation has the form

$$
\left\langle\left.\nabla G\right|_{c_{k}}, \partial \boldsymbol{c}_{k} / \partial c_{1}\right\rangle \dot{c}_{1}=\left\langle\left.\nabla G\right|_{c_{k}}, \boldsymbol{J}\left(\boldsymbol{c}_{k}\right)\right\rangle .
$$

Here $\left[\left.\nabla G\right|_{c_{k}}\right]_{i}=\ln \left[c_{k i} / c_{i}^{\mathrm{eq}}\right]$.

Analytic results were compared with the results of the numerical integration. The following set of parameters was used:

$$
\begin{aligned}
& k_{1}^{+}=1.0, \quad k_{1}^{-}=0.5, \quad k_{2}^{+}=0.4, \quad k_{2}^{-}=1.0, \\
& c_{1}^{\mathrm{eq}}=0.5, \quad c_{2}^{\mathrm{eq}}=0.1, \quad c_{3}^{\mathrm{eq}}=0.1, \quad c_{4}^{\mathrm{eq}}=0.4 \text {, } \\
& B_{1}=1.0, \quad B_{2}=0.2 \text {. }
\end{aligned}
$$

Direct numerical integration of the system has demonstrated that the manifold $c_{3}=c_{3}^{\text {eq }}$ in the plane $\left(c_{1}, c_{3}\right)$ attracts all individual trajectories. Thus, the reduced description in this example should extract this manifold.

Fig. 1 demonstrates the quasi-equilibrium manifold (45) and first two corrections found analytically. It is apparent that while the initial quasi-equilibrium approximation is in a poor agreement with the reduced description, the corrections rapidly improve the situation. This confirms our expectation of an advantage of using iteration methods in comparison to methods based on a small parameter expansions.

In the second test, we have used a different initial approximate reduced description for the same system. The initial manifold was chosen as the quasi-stationary manifold of the first reaction, i.e. it was found from an equation $W_{1}(\boldsymbol{c})=0$ :

$$
\begin{aligned}
& c_{01}=c_{1}, \\
& c_{02}=\frac{B_{2} k_{1}^{-}}{k_{1}^{-}+k_{1}^{+} c_{1}}, \\
& c_{03}=\frac{k_{1}^{+} B_{2} c_{1}}{k_{1}^{-}+k_{1}^{+} c_{1}}, \\
& c_{04}=B_{1}-c_{1}-\frac{k_{1}^{+} B_{2} c_{1}}{k_{1}^{-}+k_{1}^{+} c_{1}} .
\end{aligned}
$$

The quasi-stationary manifold (52) is parameterized by the variable $c_{1}$, and we have used again the same projectors (46) and (50) in the course of finding corrections to the manifold (52). It should be stressed that projector (46) is not thermodynamic for the quasi-stationary manifold (52) because the latter is not the quasi-equilibrium 


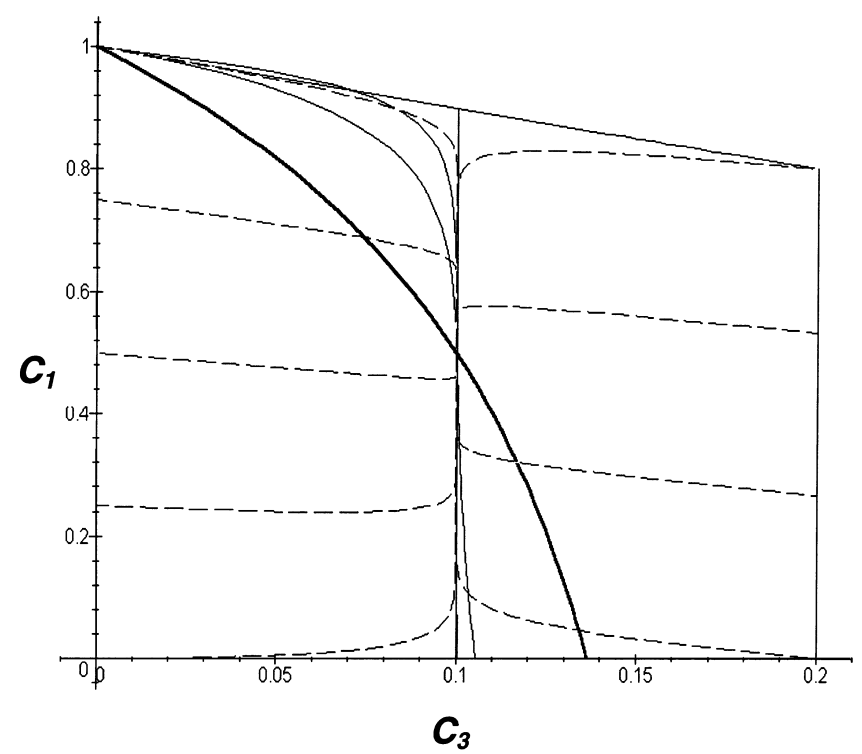

Fig. 1. Images of the initial quasi-equilibrium manifold (bold line) (44) and the first two corrections (solid normal lines) in the phase plane $\left[c_{1}, c_{3}\right]$ for two-step catalytic reaction (40). Dashed lines are individual trajectories.

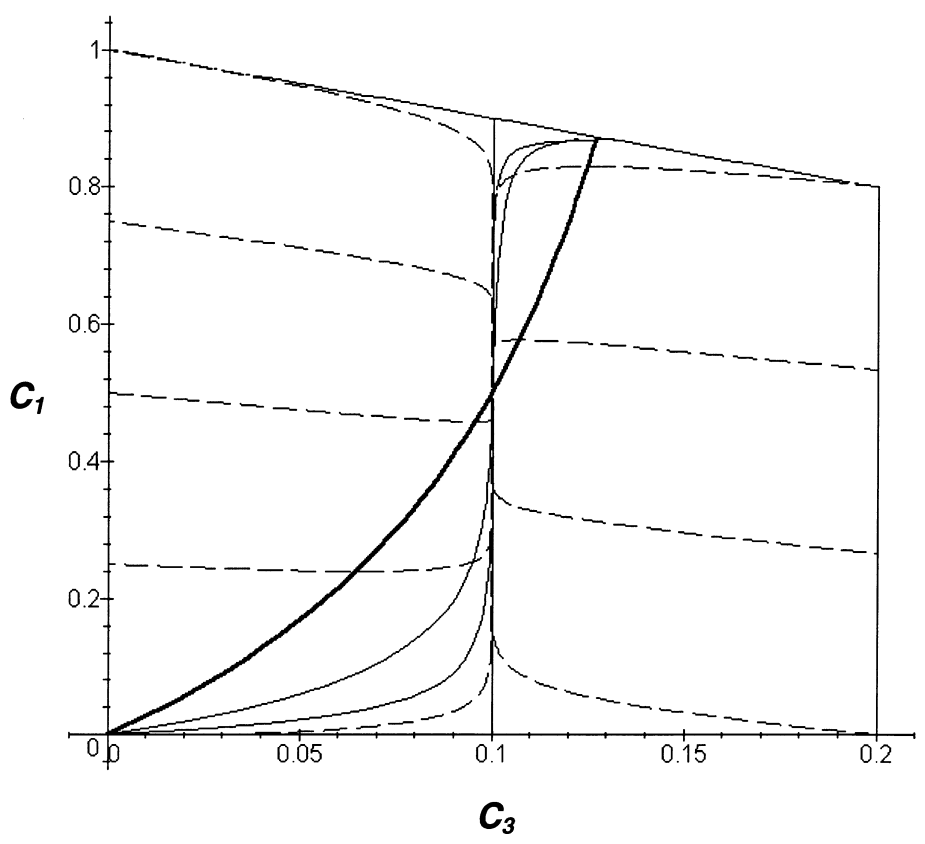

Fig. 2. Images of the initial quasi-stationary manifold (bold line) and of the first two corrections (solid normal lines) for the same system as in the Fig. 1. 


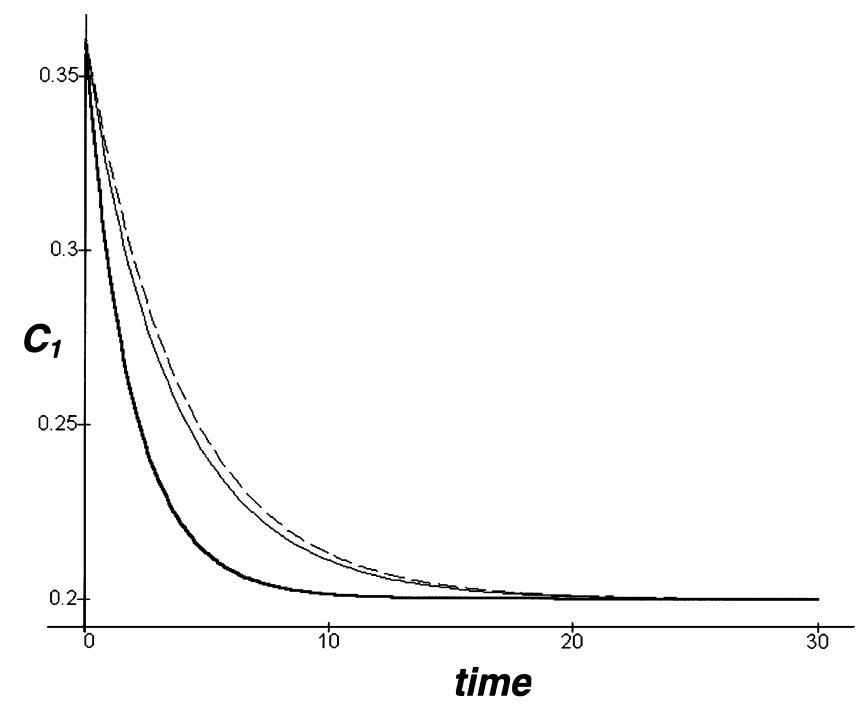

Fig. 3. Time evolution of the concentration of the specie $c_{1}$ calculated using the Eq. (51). Bold line: The initial quasi-equilibrium approximation (44). Solid line: The first correction. Dashed line: The numerical solution of the Eq. (40) corresponding to the trajectory of the slowest relaxation.

manifold. Rather, dynamics on the manifold (52) should be derived within its thermodynamic re-parameterization, that is, from the Eq. (51) for $k=0$.

In Fig. 2 we demonstrate the quasi-stationary manifold (52), together with the two first corrections. Same as in the first test, the convergency is apparent. It is important to notice here the insensitivity of the corrections to the choice of the initial approximation: While both the initial approximations (45) and (52) are poor, and differ considerably from each other, the invariant manifold is approximated fairly well after two iterations starting from either approximation.

In Fig. 3 we compare the time evolution of the concentration $c_{1}$ computed in three different ways: Integrating numerically the Eq. (51) for the quasi-equilibrium manifold (45), and for the second correction, and integrating the kinetic equation (40) with the initial condition $c_{3}(0)=c_{3}^{\mathrm{eq}}, c_{1}(0)=B_{2}-c_{3}^{\mathrm{eq}}$ which corresponds to the 'true' invariant manifold. The comparison is fairly good.

\section{Discussion}

In this paper, we have considered applications of the method of invariant manifold to an important problem of dissipative chemical kinetics: The construction of reduced description for spatially uniform reacting systems. The method of invariant manifold is extended with the symmetric linearization procedure. The technical advantage of this linearization is a simplification of the correction step. We have also given a plausible argument of its relevancy to the decomposition of motions. The method was verified 
with an example of a nonlinear reaction mechanism, and, in particular, insensitivity to the choice of the initial approximation has been demonstrated.

The following clarification is in order: The use of projection operators within the present framework should not be confused with the family of methods of nonequilibrium statistical mechanics known under the common title of projection operator formalism [12]. The fundamental difference is that in the projection operator formalism one considers individual trajectories, and starts with a formal solution of the dynamic equation which makes it applicable solely to linear equations (basically, to the Liouville equation, or, in the case of dissipative systems, to linearized kinetic equations such as the linearized Boltzmann equation). Otherwise stated, in the projection operator formalism, one attempts to construct the finals of the dynamics by tracing trajectories from their beginning, while in the method of invariant manifold one attempts to proceed in an opposite direction by choosing an ansatz for the final dynamics and then iteratively correcting it due to dynamics. In both cases, certain hypothesis are necessary in order to proceed: In the projection operator formalism, the idea of a rapid decay of memory is most important, while in the method of invariant manifold the thermodynamic and kinetic description of the decomposition of motions are essential.

A further comment concerns a generalization of the symmetric linearization procedure to construction of reduced description in other systems. In a recent series of publications $[13,14]$, it has been demonstrated that many kinetic systems can be cast in the following suggestive form:

$$
\frac{\mathrm{d} x}{\mathrm{~d} t}=L(x) \frac{\delta E}{\delta x}+M(x) \frac{\delta S}{\delta x},
$$

where $x$ is a state variable, $L$ and $M$ are operators acting linearly on the gradient of the energy $E$ and of the entropy $S$. Only the second term in the right-hand side of Eq. (53) is relevant to our discussion, and we recall that the operator $M$ is symmetric and nonnegative definite. Here we mention that the Marcelin-De Donder form of reaction kinetic equation can be recast into a similar expression: For simplicity, let us assume the detail balance condition (13). Multiplying and dividing each term in the sum (11) by the affinity, $\left\langle\gamma_{s}, \nabla G\right\rangle$, we have

$$
\boldsymbol{J}=-\boldsymbol{M} \nabla G,
$$

where the nonnegative definite symmetric matrix $\boldsymbol{M}$ has the following explicit form:

$$
\boldsymbol{M}=\sum_{s=1}^{r}\left|\boldsymbol{\gamma}_{s}\right\rangle \frac{\varphi_{s}^{+}\left\{\exp \left[\left\langle\nabla G, \boldsymbol{\alpha}_{s}\right\rangle\right]-\exp \left[\left\langle\boldsymbol{\nabla} G, \boldsymbol{\beta}_{s}\right\rangle\right]\right\}}{\left\langle\boldsymbol{\nabla} G, \boldsymbol{\alpha}_{s}\right\rangle-\left\langle\boldsymbol{\nabla} G, \boldsymbol{\beta}_{s}\right\rangle}\left\langle\boldsymbol{\gamma}_{s}\right| .
$$

This representation of the Marcelin-De Donder kinetics is relevant to the dissipative part of the Eq. (53) (moreover, it is directly relevant to a representation of the Boltzmann collision operator found in Ref. [15]). The form (54a) can be used as an alternative starting point in order to obtain a symmetric linearization around the state $\boldsymbol{c}_{0}$ : Evaluating the $\boldsymbol{c}$-dependent matrix $(54 \mathrm{~b})$ in the state $\boldsymbol{c}_{0}$, and using second-order expansion of the function $G$ around $\boldsymbol{c}_{0}$, we have

$$
\left.\boldsymbol{J}\left(\boldsymbol{c}_{0}+\delta \boldsymbol{c}\right) \approx \boldsymbol{J}\left(\boldsymbol{c}_{0}\right)+\boldsymbol{M}_{\boldsymbol{c}_{0}}|\delta \boldsymbol{c}\rangle\right\rangle .
$$


If we now consider the relaxation equation, $\left.\dot{\delta} \boldsymbol{c}=\boldsymbol{M}_{\boldsymbol{c}_{0}}|\delta \boldsymbol{c}\rangle\right\rangle$, it becomes apparent that it models the relaxation to the state $\boldsymbol{c}_{0}$ in the same sense as the relaxation equation (33). Therefore, the matrix $\boldsymbol{M}_{\boldsymbol{c}_{0}}$ could be used in the symmetric linearization procedure instead of the matrix $\boldsymbol{L}_{c_{0}}^{\prime}$ in the Eq. (34a), and therefore an extension of our formalism is possible to systems of the form (53) where the nonnegative definite symmetric operator $M$ may have no additional Marcelin-De Donder structure. It should be stressed here that though the symmetric linearization procedure is not unique, the fixed point of the correction procedure (the invariant manifold) does not depend on which linearization was used, though the corrections may approach the limit along a sequence of approximations which does depend on the linearization.

Finally, we would like to emphasize that the construction of invariant manifolds is relevant also to open systems. In that case, the most important question concerns the construction of initial approximations and derivation of the induced dynamics since these points are not supported by thermodynamic considerations anymore. An answer to these questions is planned for a subsequent publication.

\section{Acknowledgements}

IVK is indebted to H.C. Öttinger and M. Grmela for discussions.

\section{References}

[1] A.N. Gorban, I.V. Karlin, Physica A 190 (1992) 393.

[2] A.N. Gorban, I.V. Karlin, Transport Theory Stat. Phys. 23 (1994) 559.

[3] A.N. Gorban, I.V. Karlin, Physica A 206 (1994) 401.

[4] A.N. Gorban, I.V. Karlin, Phys. Rev. Lett. 77 (1996) 282.

[5] I.V. Karlin, G. Dukek, T.F. Nonnenmacher, Phys. Rev. E 55 (1997) 1573.

[6] G.S. Yablonskii, V.I. Bykov, A.N. Gorban, V.I. Elokhin, Kinetic models of catalytic reactions. Comprehensive Chemical Kinetics, Vol. 32, Elsevier, Amsterdam, 1991.

[7] A.N. Gorban, Obkhod Ravnovesiya [Equilibrium Encircling], Nauka, Novosibirsk, 1984.

[8] T. de Donder, P. Van Rysselberghe, Thermodynamic Theory of Affinity. A book of principles, University Press, Stanford, 1936.

[9] M. Feinberg, Arch. Rational Mech. Anal. 46 (1972) 1.

[10] V.I. Bykov, A.N. Gorban, G.S. Yablonskii, Reaction Kinetics Catal. Lett. 20 (1982) 261.

[11] G. Dukek, I.V. Karlin, T.F. Nonnenmacher, Physica A 239 (1997) 493.

[12] H. Grabert, Projection Operator Techniques in Nonequilibrium Statistical Mechanics, Springer, Berlin, 1982.

[13] M. Grmela, H.C. Öttinger, Phys. Rev. E 56 (1997) 6620.

[14] H.C. Öttinger, M. Grmela, Phys. Rev. E 56 (1997) 6633.

[15] H.C. Öttinger, J. Non-Equilib. Thermodyn. 22 (1997) 386. 